\title{
Study of the reactivity of squarylferrocenes. Addition of amines and aminoesters
}

\author{
Fabián Villanueva-García, ${ }^{a}$ Priscilla Reyes, ${ }^{b}$ Keith H. Pannell, ${ }^{b}$ \\ Alejandro Álvarez-Hernández, ${ }^{\mathrm{c}}$ Ismael Valois, ${ }^{\mathrm{c}}$ Juan M. Juárez-Ruiz, ${ }^{\mathrm{a}}$ \\ Juan O. Flores-Rizo, ${ }^{a}$ and Eduardo Peña-Cabrera ${ }^{a *}$ \\ ${ }^{a}$ Departamento de Química, Universidad de Guanajuato, Col. Noria Alta S/N \\ Guanajuato, GTO 36050, Mexico \\ ${ }^{b}$ Chemistry Department, University of Texas at El Paso. El Paso, TX 79968-0513, USA \\ ${ }^{c}$ Centro de Investigaciones Químicas, Universidad Autónoma del Estado de Hidalgo. Carretera \\ Pachuca-Tulancingo Km. 4.5. Cd. Universitaria, Mineral de la Reforma, Hidalgo 42076, Mexico \\ E-mail:eduardop@quijote.ugto.mx
}

\begin{abstract}
The reactivity of squarylferrocenes was studied in the addition of several $\mathrm{N}$-centered nucleophiles. A total of seven simple amines (primary and secondary) were added to both monosquarylferrocene and 1,1'-bissquarylferrocene to generate 14 ferrocenylsquaramides in 85$98 \%$. Likewise, five aminoesters were added to the same scaffolds to produce a novel family of 10 ferrocene-aminoester conjugates in good to excellent yields (57-95\%).
\end{abstract}

Keywords: Ferrocene, squaryl esters, cyclobutenediones, $\alpha$-aminoesters

\section{Introduction}

After over fifty years of its discovery, ferrocene has become the starting material for the preparation of compounds with applications in different fields. ${ }^{1}$ One emergent area in which ferrocene is playing already a leading role is bioorganometallic chemistry. ${ }^{2}$ Some of the features that render ferrocene a very attractive candidate for applications in bioorganometallic chemistry include: its electroneutrality, chemical stability, redox properties, and non-toxicity. ${ }^{3}$ As a result, a large number of reports have appeared in the literature dealing with the synthesis and biological activity of ferrocene bioconjugates. ${ }^{4}$ One example that stands out with respect to its antiproliferative activity on mammary tumors is a family of ferrocene-containing Tamoxifen analogues called Ferrocifen (Figure 1). ${ }^{5}$

Similarly, another class of important bioorganometallic compounds with significant medical applications is that of metal-peptide bioconjugates. Some of them have displayed interesting 
properties such as antiproliferative and anti-bacterial activity. ${ }^{6}$ Within this type of compounds, a great deal of efforts has focused on the synthesis and study of the properties of ferrocenepeptides.

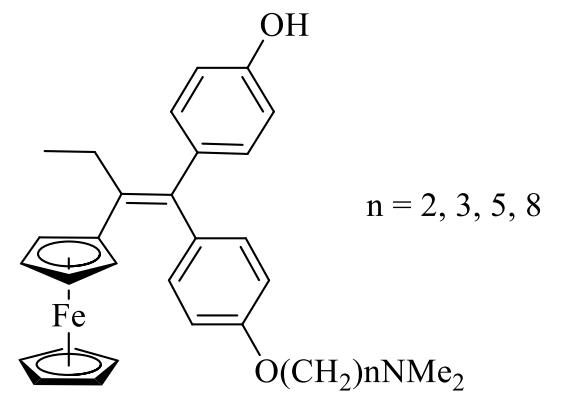

Figure 1. General structure of the Ferrocifen family.

These derivatives are important because they incorporate a redox-active moiety which is, at the same time, a scaffold that may hold two peptide strands within H-bonding distance. Several research groups have disclosed the synthesis of ferrocene-peptides and described their use as: electrochemical biosensors, ${ }^{7}$ redox-switching centers, etc. ${ }^{8}$

A different organic fragment that has been attached to peptides is squaric acid or its derivatives. Squaric acid $\mathbf{1}$ is a very attractive molecule in its own right. It has been used in medicinal chemistry, ${ }^{9}$ material science, ${ }^{10}$ and organic synthesis. ${ }^{11}$

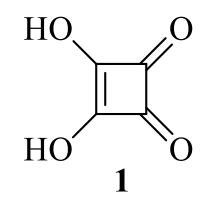

Squaric acid-peptide hybrids have been incorporated to protein conjugates which mimic the polysaccharides found on the cell surfaces of different pathogens. ${ }^{12}$ In another example, these compounds have been utilized as inhibitors of Matrix Metalloprotease $\mathbf{1} .^{13}$

Our group has disclosed the synthesis of squarylferrocenes $\mathbf{2}$ and $\mathbf{3}^{14}$ and shortly after that, building on our results, Zora et al. reported the synthesis of ferrocenylquinones from $\mathbf{2}{ }^{15}$

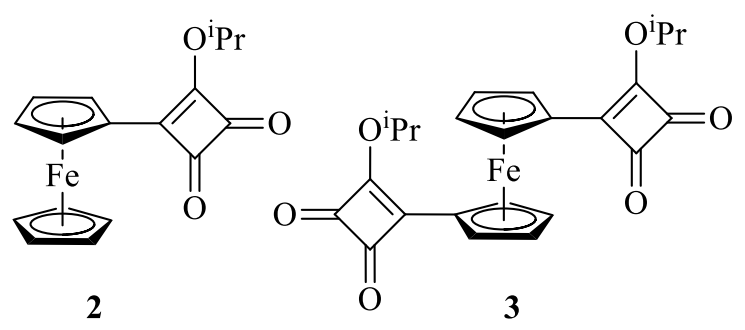


As can be appreciated, $\mathbf{2}$ and $\mathbf{3}$ already possess the two fragments whose attractive properties were aforementioned. We now report the initial study of the reactivity of these scaffolds with simple amines and aminoesters which can help pave the way for the preparation of more elaborate organometallic peptides.

The study began with the addition of simple amines to assess the reactivity of both $\mathbf{2}$ and $\mathbf{3}$ under mild conditions. ${ }^{16}$ The survey was carried out with the amines shown in Figure 2.<smiles>C1CCNCC1</smiles>

4<smiles>C1COCCN1</smiles>

5<smiles>C1CNCCN1</smiles>

6
7<smiles>NCc1ccco1</smiles>

8<smiles>NCc1ccccc1</smiles>

9
$\mathrm{MeNH}_{2}$

10

Figure 2. Amines used for reaction with $\mathbf{2}$ and $\mathbf{3}$.

We were pleased with the excellent reactivity displayed by both $\mathbf{2}$ and $\mathbf{3}$. The results are illustrated in Table 1.

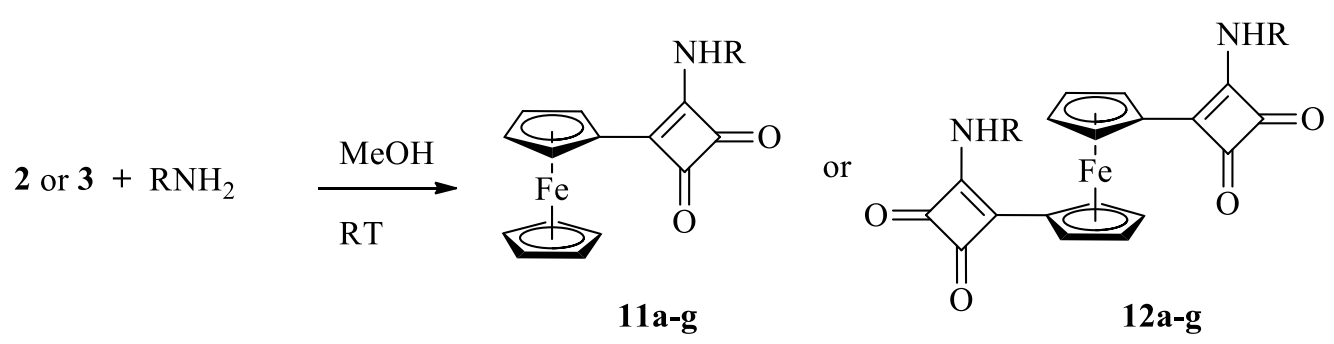

Table 1. Addition of amines 4-10 to squarylferrocenes $\mathbf{2}$ and $\mathbf{3}$

\begin{tabular}{ccccc}
\hline Entry & Amine & Product & Time & $\%$ yield $^{\mathrm{a}}$ \\
\hline 1 & $\mathbf{4}$ & $\mathbf{1 1 a}$ & $1 \mathrm{~h}$ & 91 \\
2 & $\mathbf{5}$ & $\mathbf{1 1 b}$ & $1 \mathrm{~h}$ & 98 \\
3 & $\mathbf{6}$ & $\mathbf{1 1 c}$ & $1 \mathrm{~h}$ & 97 \\
4 & $\mathbf{7}$ & $\mathbf{1 1 d}$ & $30 \mathrm{~min}$ & 93 \\
5 & $\mathbf{8}$ & $\mathbf{1 1 e}$ & $35 \mathrm{~min}$ & 85 \\
6 & $\mathbf{9}$ & $\mathbf{1 1}$ & $45 \mathrm{~min}$ & 96 \\
7 & $\mathbf{1 0}$ & $\mathbf{1 1 g}$ & $40 \mathrm{~min}$ & 95 \\
8 & $\mathbf{4}$ & $\mathbf{1 2 a}$ & $3 \mathrm{~h}$ & 95 \\
9 & $\mathbf{5}$ & $\mathbf{1 2 b}$ & $3 \mathrm{~h}$ & 97 \\
10 & $\mathbf{6}$ & $\mathbf{1 2 c}$ & $2 \mathrm{~h}$ & 80 \\
\hline
\end{tabular}


Table 1. Continued

\begin{tabular}{ccccc}
\hline Entry & Amine & Product & Time & $\%$ Yield $^{\mathrm{a}}$ \\
\hline 11 & $\mathbf{7}$ & $\mathbf{1 2 d}$ & $2 \mathrm{~h}$ & 94 \\
12 & $\mathbf{8}$ & $\mathbf{1 2 e}$ & $2 \mathrm{~h}$ & 93 \\
13 & $\mathbf{9}$ & $\mathbf{1 2 f}$ & $2 \mathrm{~h}$ & 97 \\
14 & $\mathbf{1 0}$ & $\mathbf{1 2 g}$ & $2 \mathrm{~h}$ & 92 \\
\hline
\end{tabular}

${ }^{a}$ Isolated yield. For the series 11a-g, a 1:2 ratio of 2:amine was used. For the series 12a-g, a 1:4 ratio of 3:amine was used.

The addition of the amines 4-10 to $\mathbf{2}$ and $\mathbf{3}$ took place smoothly approaching quantitative yields in several cases. The ${ }^{\mathrm{P} r O}$ - group in the squarylferrocenes showed excellent reactivity as leaving group in this addition-elimination process (Figure 3).
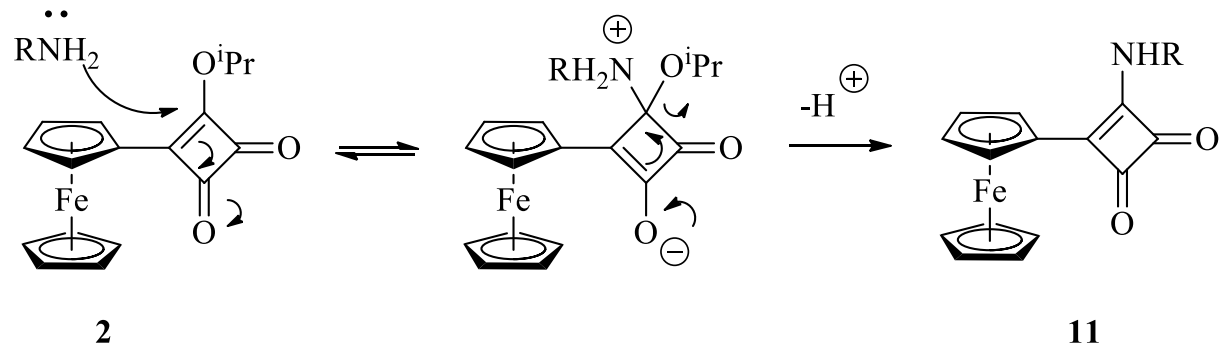

Figure 3. Addition/elimination process that gives rise to squaramides.

As expected on the basis of steric hindrance, primary amines reacted more rapidly than secondary ones. However, the yield did not decomposedrease. This trend was observed in both 11a-g and 12a-g series. Due to the fact that an excess of the amines were used in all cases, no evidence of the double addition product was observed in the case of piperazine in the ${ }^{1} \mathrm{H}$ NMR spectrum of the crude material (entries 3 and 10). No inert atmosphere was required and the reactions took place at room temperature. All of the products were deep red solids that could be purified by simply triturating the crude material with hexanes and ethyl ether.

Next, we turned our attention to the addition of aminoesters to squarylferrocenes $\mathbf{2}$ and $\mathbf{3}$. The aminoester hydrochlorides used are shown in Figure 4. 
<smiles>COC(=O)C(C)N</smiles>

13<smiles>CCOC(=O)[C@H](N)C(C)C</smiles>

14<smiles>CCOC(=O)CC[C@H](N)C(=O)OCC</smiles>

16<smiles>COC(=O)[C@H](N)CC(C)C</smiles>

15<smiles>CCOC(=O)CCN</smiles>

17

Figure 4. Aminoester hydrochlorides added to squarylferrocenes $\mathbf{2}$ and $\mathbf{3}$.

The results of this study are displayed in Table 2 .<smiles>NCCC(=O)C[Hg]Cl</smiles>

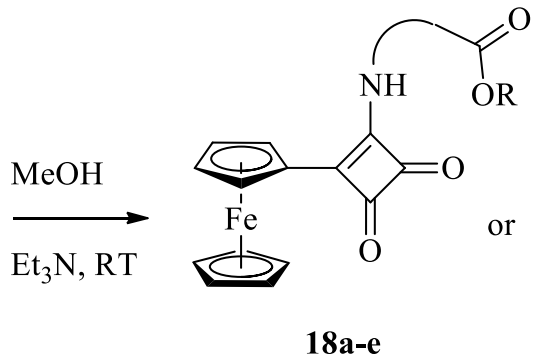<smiles></smiles>

Table 2. Addition of aminoester hydrochlorides 13-17 to squarylferrocenes 2 and 3

\begin{tabular}{ccccc}
\hline Entry & Aminoester & Product & Time $(\mathrm{h})$ & \% Yield \\
\hline 1 & $\mathbf{1 3}$ & $\mathbf{1 8 a}$ & 4 & 79 \\
2 & $\mathbf{1 4}$ & $\mathbf{1 8 b}$ & 4 & 81 \\
3 & $\mathbf{1 5}$ & $\mathbf{1 8 c}$ & 4 & 77 \\
4 & $\mathbf{1 6}$ & $\mathbf{1 8 d}$ & 4 & 78 \\
5 & $\mathbf{1 7}$ & $\mathbf{1 8 d}$ & 1.5 & 95 \\
6 & $\mathbf{1 3}$ & $\mathbf{1 9 a}$ & 24 & 57 \\
7 & $\mathbf{1 4}$ & $\mathbf{1 9 b}$ & 24 & 68 \\
8 & $\mathbf{1 5}$ & $\mathbf{1 9 c}$ & 24 & 80 \\
9 & $\mathbf{1 6}$ & $\mathbf{1 9 d}$ & 24 & 73 \\
10 & $\mathbf{1 7}$ & $\mathbf{1 9}$ & 2 & 88 \\
\hline
\end{tabular}

${ }^{a}$ Isolated yield. For the series 18a-e, a 1:2:2 ratio of 2:aminoester:TEA was used. For the series 19a-e, a 1:4:4 ratio of 3:aminoester:TEA was used.

Commercially available aminoester hydrochlorides 13-17 were added to both $\mathbf{2}$ and $\mathbf{3}$. The reaction conditions were basically the same as those specified in Table 1, except for the addition 
of a stoichiometric amount of $\mathrm{Et}_{3} \mathrm{~N}$ to liberate the aminoester. The yields were somewhat lower than the case of the addition of simple amines, however, they ranged from acceptable to good. Except for the case of $\beta$-alanine (entry 5), an increase in the reaction time was observed in all cases, especially in the addition of the aminoesters to the bis-squarylferrocene 3 . This general observation may be attributed to the increased steric bulk at the position adjacent to the amino group in the aminoesters. As in the case of the addition of simple amines, products 18-19 were dark red solids that were purified by simple trituration of the crude material with hexanes and ethyl ether.

Ferrocenyl derivatives 18a-e and 19a-e, once hydrolyzed to the corresponding free carboxylic acids, may be used to incorporate other aminoacids or peptide chains.

\section{Conclusions}

Squarylferrocenes $\mathbf{2}$ and $\mathbf{3}$ exhibited excellent reactivity toward the addition of both primary and secondary amines. Likewise, aminoester conjugates were prepared in the same fashion in high yields. The possibility to react free amino oligopeptides directly to $\mathbf{2}$ and $\mathbf{3}$ in a convergent manner is being currently investigated in our group and will be disclosed in due course.

\section{Experimental Section}

General. ${ }^{1} \mathrm{H}$ NMR spectra were recorded on a Varian Gemini 200 (200 MHz) in DMSO-d6 (2.49 ppm) as internal reference unless otherwise indicated. Data are reported in the following order: chemical shift in ppm $(\delta)$, multiplicities (br (broadened)), s (singlet), $\mathrm{d}$ (doublet), $\mathrm{t}$ (triplet), q (quartet), sex (sextet), hep (heptet), m (multiplet), exch (exchangeable), app (apparent), coupling constants, $J$, are reported $(\mathrm{Hz})$, and integration. Infrared spectra were recorded on a Perkin-Elmer FTRI 1600 series spectrophotometer. Peaks are reported $\left(\mathrm{cm}^{-1}\right)$ with the following relative intensities: s (strong 67-100\%), m (medium 40-67\%), and w (weak 20$40 \%)$.

Analytical thin-layer chromatography was performed on Merck silica gel plates with F-254 indicator. Accurate mass spectra were obtained on a Bruker microTOF fitted with an ESI.

\section{General procedure for the synthesis of ferrocene monosquaramides (11a-g)}

A $20-\mathrm{mL}$ vial was charged with 2 (50 mg, $0.15 \mathrm{mmol}, 1$ equiv), the corresponding amine $(0.30$ mmol, 2 equiv), methanol ( $4 \mathrm{~mL})$, and a magnetic stirrer. The vial was capped and the mixture was stirred until completion whereupon the solvent was removed under reduced pressure. The product was purified by simply trituration with hexanes and ethyl ether. 


\section{General procedure for the synthesis of ferrocene bissquaramides (12a-g)}

A $20-\mathrm{mL}$ vial was charged with $\mathbf{3}^{14 \mathrm{~b}}$ (50 $\mathrm{mg}, 0.11 \mathrm{mmol}, 1$ equiv), the corresponding amine $(0.43$ mmol, 4 equiv), methanol ( $4 \mathrm{~mL})$, and a magnetic stirrer. The vial was capped and the mixture was stirred until completion whereupon the solvent was removed under reduced pressure. The product was purified by simply trituration with hexanes and ethyl ether.

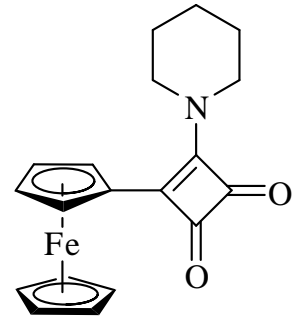

3-Ferrocenyl-4-(piperidin-1-yl)cyclobut-3-ene-1,2-dione (11a). Red solid (48 mg, $91 \%$ ). TLC ( $\mathrm{R}_{f}=0.7,90 \%$ AcOEt/hexanes); mp: $262{ }^{\circ} \mathrm{C}$ (decomposed); IR (KBr, cm $\left.{ }^{-1}\right): 2937$ (m), 2869 (m), 1772 (i), 1716 (s), 1603 (s), 1495 (s), 1454 (m), 1357 (m), 1032 (m), 934 (m). ${ }^{1} \mathrm{H}$ NMR (200 MHz, DMSO- $d_{6}$ ): $\delta 4.7$ (app t, $J=1.8 \mathrm{~Hz}, 2 \mathrm{H}$ ), 4.6 (app t, $J=1.8,2 \mathrm{H}$ ), 3.9 (br, 2H), 3.8 (br, 2H), 1.6 (br, $6 \mathrm{H}) .{ }^{13} \mathrm{C}$ NMR (50 MHz, DMSO- $d_{6}$ ): 191.9, 188.1, 175.5, 165.1, 79.1, 70.9. 69,7, 68.9, 25.2, 23.0. HRMS FABS $(\mathrm{M}+\mathrm{H})^{+}$Calcd for $\mathrm{C}_{19} \mathrm{H}_{20} \mathrm{FeNO}_{2}$ : 350.0843. Found: 350.0844. Anal. Calcd for $\mathrm{C}_{19} \mathrm{H}_{19} \mathrm{FeNO}_{2}$ : C, 65.35; H, 5.48; Fe, 15.99; N, 4.01; O, 9.16. Found C, 65.63 ; H, 5.81; N, 3.94.

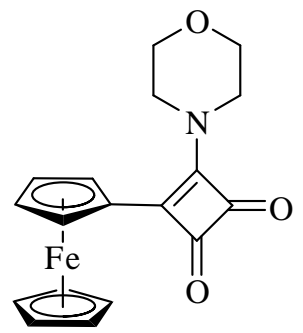

3-Ferrocenyl-4-morpholino-cyclobut-3-ene-1,2-dione (11b). Red solid (51 mg, 98\%). TLC ( $\mathrm{R}_{f}$ = 0.7, 90\% AcOEt/hexanes); $\mathrm{mp}>242{ }^{\circ} \mathrm{C}$ (decomposed); IR (KBr): 2918 (m), 1771 (i), 1720 (s), 1604 (s), 1498 (i), 1450 (s), 1309 (i)1287 (s), 1022, (m), 909 (m). ${ }^{1}$ H NMR (200 MHz, DMSO$\left.d_{6}\right): \delta 4.7$ (br s, 2H), 4.6 (br s, 2H), 4.2 (s, 5H), 3.9 (br, 4H), 3.8 (br, 4H). ${ }^{13} \mathrm{C} \mathrm{NMR} \mathrm{(50} \mathrm{MHz,}$ DMSO-d6): 191.6, 188.1, 175.7, 165.5, 71.0, 70.5, 69.7, 69.1, 65.6. HRMS FABS (M+H) ${ }^{+}$Calcd for $\mathrm{C}_{18} \mathrm{H}_{18} \mathrm{FeNO}_{3}$ : 352.0636. Found: 352.0638. Anal. Calcd for $\mathrm{C}_{18} \mathrm{H}_{17} \mathrm{FeNO}_{3}$ : C, 61.56; H, 4.88; Fe, 15.90; N, 3.99; O, 13.67. Found C, 61.66 ; H, 4.95; N, 3.88. 


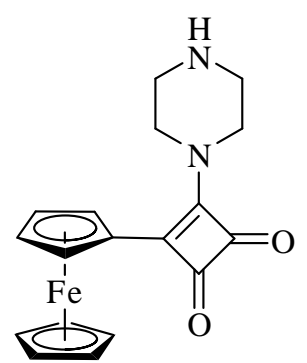

3-Ferrocenyl-4-(piperazin-1-yl)cyclobut-3-ene-1,2-dione (11c). Red solid (51 mg, 97\%). TLC $\left(\mathrm{R}_{f}=0.4,2 \% \mathrm{MeOH} / \mathrm{CH}_{2} \mathrm{Cl}_{2}\right)$; mp: $285^{\circ} \mathrm{C}$ (decomposed); IR (KBr, cm $\left.{ }^{-1}\right): 3320(\mathrm{~m}), 2359(\mathrm{~m})$, 1770 (s), 1711 (s), 1602 (m), 1498 (m), 1270 (m), 1135 (m), 1006 (m), 817 (m). ${ }^{1} \mathrm{H}$ NMR (200 MHz, DMSO- $\left.d_{6}\right): \delta 4.7$ (br, 2H), $4.6(\mathrm{br}, 2 \mathrm{H}), 4.2(\mathrm{~s}, 5 \mathrm{H}), 3.8(\mathrm{br}, 4 \mathrm{H}), 2.8(\mathrm{br}, 4 \mathrm{H}) .{ }^{13} \mathrm{C} \mathrm{NMR}$ (50 MHz, DMSO-d6): 191.8, 188.0, 175.4, 165.2, 70.9, 70.8, 69.7, 69.1, 49.6, 46.9, 45.4. HRMS FABS $(\mathrm{M}+\mathrm{H})^{+}$Calcd for $\mathrm{C}_{18} \mathrm{H}_{19} \mathrm{FeN}_{2} \mathrm{O}_{2}$ : 351.0796. Found: 351.0789. Anal. Calcd for $\mathrm{C}_{18} \mathrm{H}_{18} \mathrm{FeN}_{2} \mathrm{O}_{2}$ : C, 61.74; H, 5.18; Fe, 15.95; N, 8.00; O, 9.14. Found C, 61.69 ; H, 4.99; N, 8.15.

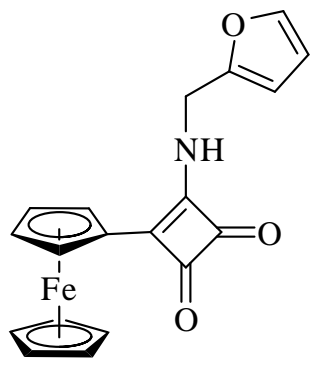

3-Ferrocenyl-4-(furan-2-ylmethyamino)cyclobut-3-ene-1,2-dione (11d). Red solid (50 mg, 93 $\%)$. TLC $\left(\mathrm{R}_{f}=0.7,90 \%\right.$ AcOEt/hexanes); $\mathrm{mp}=222^{\circ} \mathrm{C}$ (decomposed); IR $\left(\mathrm{KBr}, \mathrm{cm}^{-1}\right): 3144(\mathrm{~m})$, 2927 (m), 1778 (s), 1712 (s), 1598 (s), 1577 (s), 1522 (s), 1423 (m), 1219 (m), 1148 (m), 1085 (m), $823(\mathrm{~m}) .{ }^{1} \mathrm{H}$ NMR (200 MHz, DMSO-d $)$ : $\delta 9.0$ (br, 1H), 7.6 (s, 1H), $6.4(\mathrm{~d}, J=9 \mathrm{~Hz}, 2 \mathrm{H})$, $5.0(\mathrm{~s}, 2 \mathrm{H}), 4.8(\operatorname{app} \mathrm{d}, J=3.2 \mathrm{~Hz}, 2 \mathrm{H}), 4.6(\mathrm{~s}, 2 \mathrm{H}), 4.14(\mathrm{~s}, 5 \mathrm{H}) .{ }^{13} \mathrm{C}$ NMR $(50 \mathrm{MHz}, \mathrm{DMSO}-$ $\left.d_{6}\right)$ : 191.6, 188.2, 177.8, 168.0, 151.3, 142.9, 110.6, 107.8, 71.4, 69.8, 67.5. HRMS FABS $(\mathrm{M}+\mathrm{H})^{+}$Calcd for $\mathrm{C}_{19} \mathrm{H}_{16} \mathrm{FeNO}_{3}: 362.0479$. Found: 362.0462. Anal. Calcd for $\mathrm{C}_{19} \mathrm{H}_{15} \mathrm{FeNO}_{3}$ : C, 63.18; H, 4.19; Fe, 15.46; N, 3.88; O, 13.29. Found C, 63.28 ; H, 4.24; N, 3.97.

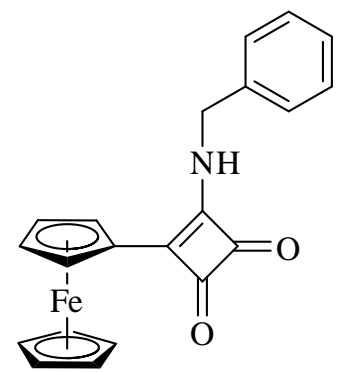


3-Benzylamino-4-ferrocenylcyclobut-3-ene-1,2-dione (11e). Red solid (47 mg, 85\%). TLC ( $\mathrm{R}_{f}$ $=0.90,90 \%$ AcOE/hexanes); $\mathrm{mp}=266^{\circ} \mathrm{C}$ (decomposed); IR (KBr, cm $\left.{ }^{-1}\right): 3109(\mathrm{~m}), 2927(\mathrm{~m})$, 1777 (s), 1706 (s), 1576 (s), 1515 (s), 1455 (m), 1440 (m), 1228 (m), 1170 (m), 1028 (m), 925 (m). ${ }^{1} \mathrm{H}$ NMR (200 MHz, DMSO-d6): $\delta 9.1$ (br, 1H), 7.3 (s, 5H), $5.1(\mathrm{~s}, 2 \mathrm{H}), 4.9(\mathrm{~d}, J=\mathrm{Hz}, 2 \mathrm{H})$, 4.6 (s, 2H), 4.2 (s, 5H). ${ }^{13} \mathrm{C}$ NMR (50 MHz, DMSO-d $)$ : 191.8, 188.0, 177.6, 167.8, 138.5, 128.6, 127.4, 127.3, 71.3, 69.9, 69.7, 67.4, 47.1. HRMS FABS $(\mathrm{M}+\mathrm{H})^{+}$Calcd for $\mathrm{C}_{21} \mathrm{H}_{18} \mathrm{FeNO}_{2}$ : 372.0687. Found: 372.0678. Anal. Calcd for $\mathrm{C}_{21} \mathrm{H}_{17} \mathrm{FeNO}_{2}$ : C, 67.95; H, 4.62; Fe, 15.04; N, 3.77; O, 8.62. Found C, $68.18 ; \mathrm{H}, 4.37 ; \mathrm{N}, 3.61$.

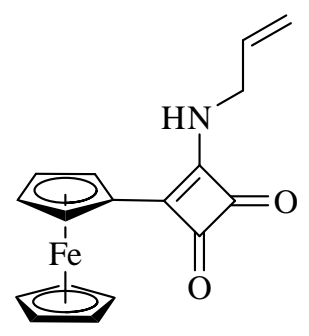

3-Allylamino-4-ferrocenylcyclobut-3-ene-1,2-dione (11f). Red solid (46 mg, 96\%). TLC ( $\mathrm{R}_{f}$ =0.8, 90\% AcOEt/hexanes); mp: $235^{\circ} \mathrm{C}$ (decomposed); IR (KBr, cm $\left.{ }^{-1}\right): 3153(\mathrm{~m}), 1777$ (s), 1709 (s), 1580 (s), 1514 (s), 1422 (m), 925.4 (m). ${ }^{1} \mathrm{H}$ NMR (200 MHz, DMSO-d6): $\delta 8.7$ (br, $\left.1 \mathrm{H}\right), 5.9$ $(\mathrm{m}, 1 \mathrm{H}), 5.2(\mathrm{~d}, J=7 \mathrm{~Hz}, 1 \mathrm{H}), 5.2(\mathrm{~s}, 1 \mathrm{H}), 5.1(\mathrm{~s}, 2 \mathrm{H}), 4.6(\mathrm{~s}, 2 \mathrm{H}), 4.3(\mathrm{br} \mathrm{s}, 2 \mathrm{H}), 4.2(\mathrm{~s}, 5 \mathrm{H}) .{ }^{13} \mathrm{C}$ NMR (50 MHz, DMSO-d6): 191.7, 188.2, 177.8, 167.5, 135.2, 116.0, 71.3, 70.0, 69.7, 67.4, 45.8. HRMS FABS (M+H) ${ }^{+}$Calcd for $\mathrm{C}_{17} \mathrm{H}_{16} \mathrm{FeNO}_{2}$ : 322.0530. Found: 322.0519. Anal. Calcd for $\mathrm{C}_{17} \mathrm{H}_{15} \mathrm{FeNO}_{2}$ : C, 63.58; $\mathrm{H}, 4.71 ; \mathrm{Fe}, 17.39 ; \mathrm{N}, 4.36 ; \mathrm{O}, 9.96$. Found $\mathrm{C}, 63.36$; H, 4.53; N, 4.33 .

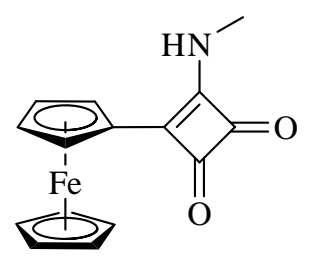

3-Ferrocenyl-4-methylaminocyclobut-3-ene-1,2-dione (11g). Red solid (42 mg, 95\%). TLC ( $\mathrm{R}_{f}=$ 0.7, 90\% AcOEt/hexanes); mp: $271{ }^{\circ} \mathrm{C}$ (decomposed); IR (KBr, cm $\left.{ }^{-1}\right): 3155$ (m), $2931(\mathrm{~m})$, 1777 (s), 1715 (s), 1586 (s), 1521 (s), 1412 (s), 1393 (s), 1003 (m), 927 (m). ${ }^{1} \mathrm{H}$ NMR (200 MHz, DMSO-d6): $\delta 8.4$ (br s, 1H), 5.0 (br s, 2H), 4.6 (br s, 2H), $4.2(\mathrm{~s}, 5 \mathrm{H}), 3.3(\mathrm{~d}, J=5,3 \mathrm{H}) .{ }^{13} \mathrm{C}$ NMR (50 MHz, DMSO-d6): 192.2, 188.1, 178.1, 167.0, 71.1, 70.2, 69.7, 67.3, 30.8. HRMS FABS $(\mathrm{M}+\mathrm{H})^{+}$Calcd for $\mathrm{C}_{15} \mathrm{H}_{14} \mathrm{FeNO}_{2}$ : 296.0374. Found: 296.0365. Anal. Calcd for $\mathrm{C}_{15} \mathrm{H}_{13} \mathrm{FeNO}_{2}$ : C, 61.05; H, 4.44; Fe, 18.92; N, 4.75; O, 10.84. Found C, 61.35; H, 4.29; N, 4.56. 


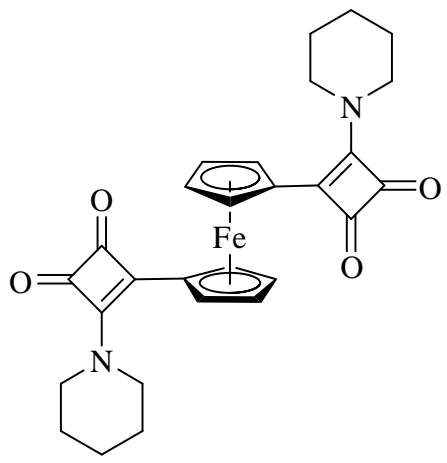

4,4'-(1,1'-Ferrocenyl)bis(3-(piperidin-1-yl)cyclobut-3-ene-1,2-dione) (12a). Red solid (52 mg, 95\%). TLC $\left(\mathrm{R}_{f}=0.2, \mathrm{CH}_{2} \mathrm{Cl}_{2} / \mathrm{MeOH} / \mathrm{CH}_{3} \mathrm{CO}_{2} \mathrm{H}, 7: 4: 1\right) ; \mathrm{mp}: 288^{\circ} \mathrm{C}$ (decomposed); IR ( $\mathrm{KBr}$, $\left.\mathrm{cm}^{-1}\right)$ : $3090(\mathrm{w}), 2945(\mathrm{~m}), 2864(\mathrm{~m}), 1769$ (s), 1722 (s), 1618 (s), 1499 (s), 1451 (s), 1397 (m), $1284(\mathrm{~m}), 1025$ (m), 933 (m). ${ }^{1} \mathrm{H}$ NMR (200 MHz, CDCl $): \delta 4.9$ (app t, $\left.J=1.8 \mathrm{~Hz}, 4 \mathrm{H}\right), 4.5$ (app t, $J=1.8 \mathrm{~Hz}, 4 \mathrm{H}), 3.9(\operatorname{app~d}, J=5.4 \mathrm{~Hz}, 4 \mathrm{H}), 3.4(\operatorname{app~d}, J=5.2 \mathrm{~Hz}, 4 \mathrm{H}), 1.7($ br s, $12 \mathrm{H})$. ${ }^{13} \mathrm{C} \mathrm{NMR}\left(50 \mathrm{MHz}, \mathrm{CDCl}_{3}\right):$ 192.5, 188.6, 177.3, 161.9, 76.0, 72.2, 70.7, 50.3, 48.3, 25.9, 25.3, 23.5. HRMS FABS $(\mathrm{M}+\mathrm{H})^{+}$Calcd for $\mathrm{C}_{28} \mathrm{H}_{29} \mathrm{FeN}_{2} \mathrm{O}_{4}: 513.1477$. Found: 513.1500. Anal. Calcd for $\mathrm{C}_{28} \mathrm{H}_{28} \mathrm{FeN}_{2} \mathrm{O}_{4}: \mathrm{C}, 65.64 ; \mathrm{H}, 5.51 ; \mathrm{Fe}, 10.90 ; \mathrm{N}, 5.47 ; \mathrm{O}, 12.49$. Found $\mathrm{C}, 65.36 ; \mathrm{H}, 5.73 ; \mathrm{N}$, 5.38 .

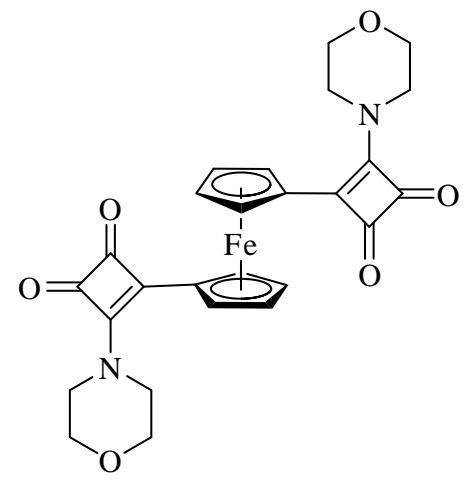

4,4'-(1,1'-Ferrocenyl)bis(3-morpholinocyclobut-3-ene-1,2-dione) (12b). Red solid (54 mg, 97\%). TLC $\left(\mathrm{R}_{f}=0.5, \mathrm{CH}_{2} \mathrm{Cl}_{2} / \mathrm{MeOH} / \mathrm{CH}_{3} \mathrm{CO}_{2} \mathrm{H}\right) ; \mathrm{mp}: 276^{\circ} \mathrm{C}$ (decomposed); $\mathrm{IR}\left(\mathrm{KBr}, \mathrm{cm}^{-1}\right)$ : 2963 (m), 2865 (m), 1774 (s), 1724 (s), 1615 (s)1498 (s), 1399 (m), 1277 (m), 1071 (m), 908 (m). ${ }^{1} \mathrm{H} \mathrm{NMR}\left(200 \mathrm{MHz}, \mathrm{CDCl}_{3}\right.$ ): $\delta 4.9$ (br s, 4H), 4.5 (br s, 4H), 4.0 (br s, 2H), 3.88 (br s, 4H), 3.5 (br s, 2H). ${ }^{13} \mathrm{C}$ NMR (50 MHz, $\mathrm{CDCl}_{3}$ ): 192.1, 188.7, 177.5, 162.3, 75.6, 72.6, 70.8, 66.7, 65.8, 49.1, 47.3. HRMS FABS $(\mathrm{M}+\mathrm{H})^{+}$Calcd for $\mathrm{C}_{26} \mathrm{H}_{25} \mathrm{FeN}_{2} \mathrm{O}_{6}$ : 517.1062. Found: 517.1032. Anal. Calcd for $\mathrm{C}_{26} \mathrm{H}_{24} \mathrm{FeN}_{2} \mathrm{O}_{6}$ : C, 60.48; H, 4.69; Fe, 10.82; N, 5.43; O, 18.59. Found C, 60.40; H, $4.71 ; \mathrm{N}, 5.48$. 


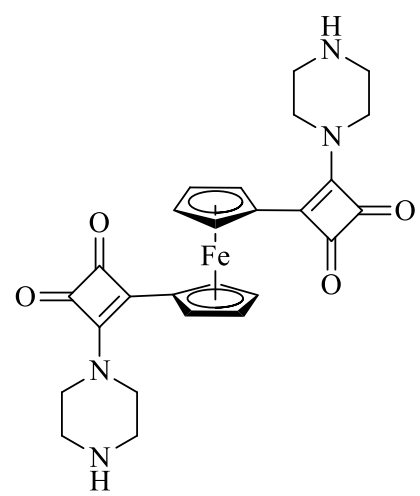

4,4'-(1,1'-Ferrocenyl)bis(3-(piperazin-1yl)cyclobut-3-ene-1,2-dione) (12c). Six equiv of piperazine were used. Red solid (44 mg, 80\%). TLC ( $\mathrm{R}_{f}=0.15, \mathrm{CH}_{2} \mathrm{Cl}_{2} / \mathrm{MeOH} / \mathrm{CH}_{3} \mathrm{CO}_{2} \mathrm{H}$, 7:4:1); mp: $247{ }^{\circ} \mathrm{C}$ (decomposed); IR (KBr, cm $\left.{ }^{-1}\right): 3344$ (w), 2921 (m), 2831 (m), 1770 (s), 1721 (s), 1615 (s), 1496 (s), 1273 (m). ${ }^{1} \mathrm{H}$ NMR (200 MHz, DMSO- $d_{6}$ ): $\delta 4.82$ (br s, 4H), 4.65 (br s, 4 H), 3.76 (br s, $4 \mathrm{H}$ ), 2.83 (br s, $10 \mathrm{H}$ ). ${ }^{13} \mathrm{C}$ NMR (50 MHz, DMSO- $d_{6}$ ): 192.3, 189.1, 176.7, 167.3, 72.2, 71.8, 70.4, 50.4, 47.9, 46.2. HRMS FABS $(\mathrm{M}+\mathrm{H})^{+}$Calcd for $\mathrm{C}_{26} \mathrm{H}_{27} \mathrm{FeN}_{4} \mathrm{O}_{4}$ : 515.1382. Found: 515.1395. Anal. Calcd for $\mathrm{C}_{26} \mathrm{H}_{26} \mathrm{FeN}_{4} \mathrm{O}_{4}$ : C, 60.71; H, 5.10; Fe, 10.86; N, 10.89; O, 12.44. Found C, 60.48; H, 5.31; N, 10.95 .

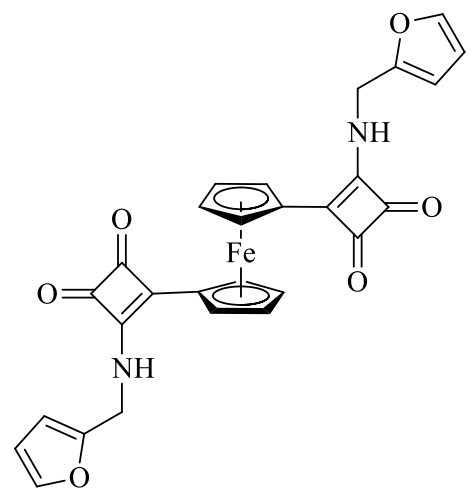

4,4'-(1,1'-Ferrocenyl)bis(3-(furan-2-ylmethylamino)cyclobut-3-ene-1,2-dione) (12d). Red solid (54 mg, 94\%). TLC ( $\left.\mathrm{R}_{f}=0.15, \mathrm{CH}_{2} \mathrm{Cl}_{2} / \mathrm{MeOH} / \mathrm{CH}_{3} \mathrm{CO}_{2} \mathrm{H}, 7: 4: 1\right)$; mp: $263{ }^{\circ} \mathrm{C}$ (decomposed); IR (KBr): 3259 (m), 2953 (m), 1775 (i), 1720 (s),1578 (s), 1515 (s), 1425 (m), $1082(\mathrm{~m}), 1012(\mathrm{~m}), 923(\mathrm{~m}), 496(\mathrm{~m}) .{ }^{1} \mathrm{H}$ NMR (200 MHz, DMSO- $\left.d_{6}\right): \delta 9.01(\mathrm{br} \mathrm{s}, 2 \mathrm{H}), 7.63(\mathrm{~s}$, 2H), $6.42(\mathrm{~s}, 4 \mathrm{H}), 5.07(\mathrm{br} \mathrm{s}, 4 \mathrm{H}), 4.76(\mathrm{~d}, J=5 \mathrm{~Hz}, 4 \mathrm{H}), 4.63(\mathrm{br} \mathrm{s}, 4 \mathrm{H}) .{ }^{13} \mathrm{C} \mathrm{NMR}(50 \mathrm{MHz}$, DMSO- $\left.d_{6}\right): 191.9,187.9,177.2,163.9,150.9,142.9,110.6,108.1,72.6,72.4,68.7$. HRMS FABS $(\mathrm{M}+\mathrm{H})^{+}$Calcd for $\mathrm{C}_{28} \mathrm{H}_{21} \mathrm{FeN}_{2} \mathrm{O}_{6}$ : 537.0749. Found: 537.0765. Anal. Calcd for $\mathrm{C}_{28} \mathrm{H}_{20} \mathrm{FeN}_{2} \mathrm{O}_{6}: \mathrm{C}, 62.71 ; \mathrm{H}, 3.76 ; \mathrm{Fe}, 10.41 ; \mathrm{N}, 5.22 ; \mathrm{O}, 17.90$. Found $\mathrm{C}, 62.66 ; \mathrm{H}, 3.79 ; \mathrm{N}$, 5.11 . 


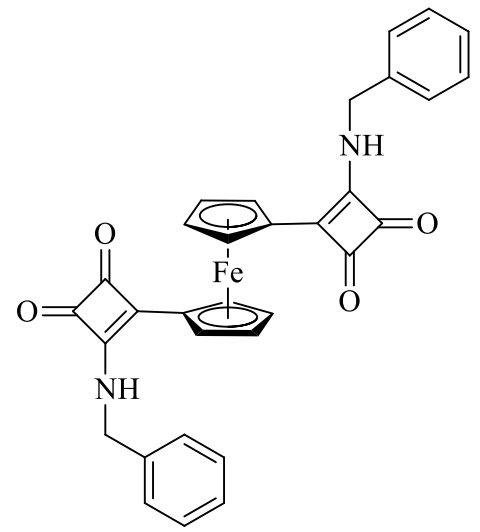

4,4'-(1,1'-Ferrocenyl)bis(3-(benzylamino)cyclobut-3-ene-1,2-dione) (12e). Violet solid (56 $\mathrm{mg}, 93 \%)$. TLC $\left(\mathrm{R}_{f}=0.2, \mathrm{CH}_{2} \mathrm{Cl}_{2} / \mathrm{MeOH} / \mathrm{CH}_{3} \mathrm{CO}_{2} \mathrm{H}, 7: 4: 1\right) ; \mathrm{mp:} 256{ }^{\circ} \mathrm{C}$ (decomposed); IR $\left(\mathrm{KBr}, \mathrm{cm}^{-1}\right): 3154(\mathrm{~m}), 3050(\mathrm{~m}), 1775(\mathrm{~m}), 1718$ (s), $1584(\mathrm{~m}), 1514(\mathrm{~m}), 1436(\mathrm{~m}) .{ }^{1} \mathrm{H}$ NMR (200 MHz, DMSO- $\left.d_{6}\right): \delta 9.0(\mathrm{t}, J=6 \mathrm{~Hz}, 2 \mathrm{H}), 7.4(\mathrm{~s}, 10 \mathrm{H}), 5.1(\mathrm{br} \mathrm{s}, 4 \mathrm{H}), 4.8(\mathrm{~d}, J=6 \mathrm{~Hz}, 4 \mathrm{H})$, 4.6 (br s, 4H). ${ }^{13} \mathrm{C}$ NMR (50 MHz, DMSO-d $d_{6}$ ): 192.3, 187.9, 177.2, 163.7, 138.2, 128.6, 127.5, 72.9, 72.4, 68.6, 47.4. HRMS FABS $(\mathrm{M}+\mathrm{H})^{+}$Calcd for $\mathrm{C}_{32} \mathrm{H}_{25} \mathrm{FeN}_{2} \mathrm{O}_{4}$ : 557.1164. Found: 557.1137. Anal. Calcd for $\mathrm{C}_{32} \mathrm{H}_{24} \mathrm{FeN}_{2} \mathrm{O}_{4}$ : C, 69.08; H, 4.35; Fe, 10.04; N, 5.03; O, 11.50. Found C, 68.75; H, 3.02; N, 4.96 .

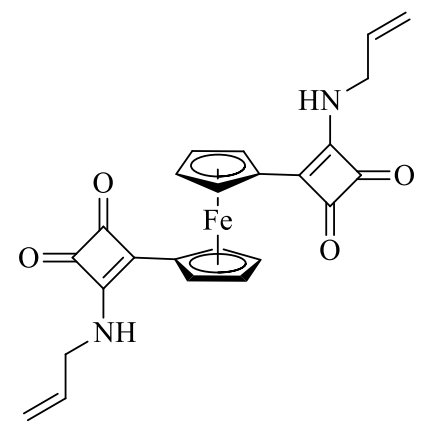

4,4'-(1,1'-Ferrocenyl)bis(3-(allylylamino)cyclobut-3-ene-1,2-dione) (12f). Red solid (47 mg, 97\%). TLC ( $\left.\mathrm{R}_{f}=0.2, \mathrm{CH}_{2} \mathrm{Cl}_{2} / \mathrm{MeOH} / \mathrm{CH}_{3} \mathrm{CO}_{2} \mathrm{H}, 7: 2: 1\right) ; \mathrm{mp}: 27{ }^{\circ} \mathrm{C}$ (decomposed); IR (KBr): 3440 (s), 3072 (m), 3008 (m), 2249 (s), 2123 (s), 1772 (s), 1715 (s), 1659 (m), 1603 (m), 1508 (m) 1226 (s), 1027 (s), 679 (s), 668 (s), $474(\mathrm{~m}) .{ }^{1} \mathrm{H}$ NMR (200 MHz, DMSO-d6): $\delta 8.7$ (t, $J=$ $6.2 \mathrm{~Hz}, 2 \mathrm{H}), 6.0(\mathrm{~m}, 2 \mathrm{H}), 5.2(\mathrm{~d}, J=11 \mathrm{~Hz}, 4 \mathrm{H}), 5.1(\mathrm{~d}, J=3.2 \mathrm{~Hz}, 4 \mathrm{H}), 5.0(\mathrm{~s}, 4 \mathrm{H}), 4.6(\mathrm{~s}, 4 \mathrm{H})$, $4.2(\mathrm{t}, J=5.4,4 \mathrm{H}) .{ }^{13} \mathrm{C}$ NMR (50 MHz, DMSO- $\left.d_{6}\right): 191.9,187.8,177.1,163.2,134.8,116.5$, 72.9, 72.1, 68.42, 46.0. HRMS FABS $(\mathrm{M}+\mathrm{H})^{+}$Calcd for $\mathrm{C}_{24} \mathrm{H}_{21} \mathrm{FeN}_{2} \mathrm{O}_{4}$ : 457.0851. Found: 457.0877. Anal. Calcd for $\mathrm{C}_{24} \mathrm{H}_{20} \mathrm{FeN}_{2} \mathrm{O}_{4}$ : C, 63.18; H, 4.42; Fe, 12.24; N, 6.14; O, 14.03. Found C, 63.28; H, 4.22; N, 6.25 . 


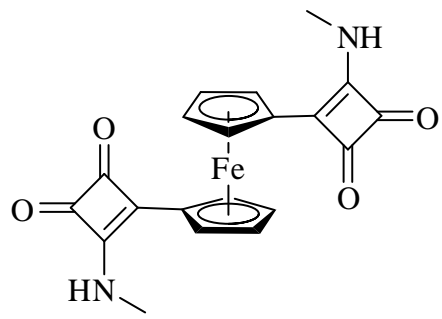

4,4'-(1,1'-Ferrocenyl)bis(3-(methylamino)cyclobut-3-ene-1,2-dione) (12g). Red solid (40 mg, 92\%). TLC ( $\left.\mathrm{R}_{f}=0.3, \mathrm{CH}_{2} \mathrm{Cl}_{2} / \mathrm{MeOH} / \mathrm{CH}_{3} \mathrm{CO}_{2} \mathrm{H}, 7: 4: 1\right)$; mp: $230{ }^{\circ} \mathrm{C}$ (decomposed); $\mathrm{IR}(\mathrm{KBr}$, $\mathrm{cm}^{-1}$ ): 3446 (s), 3070 (s), 3009 (s), 1772 (s), 1715 (s), 1654 (m), 1617 (m), 1514 (m), 1057 (s), 1028 (s), 1008 (s), 760 (m), 474 (m). ${ }^{1} \mathrm{H}$ NMR (200 MHz, DMSO-d $\left.d_{6}\right): \delta 8.32$ (d, $\left.J=4.6 \mathrm{~Hz}, 2 \mathrm{H}\right)$, 5.02 (br s, 4H), 4.64 (br s, 4H), 3.142 (d, $J=4.6 \mathrm{~Hz}, 6 \mathrm{H}) .{ }^{13} \mathrm{C}$ NMR (50 MHz, DMSO-d 6 ): 192.5, 187.9, 177.2, 162.2, 73.7, 72.1, 68.2, 30.7. HRMS FABS $(\mathrm{M}+\mathrm{H})^{+}$Calcd for $\mathrm{C}_{20} \mathrm{H}_{17} \mathrm{FeN}_{2} \mathrm{O}_{4}$ : 405.0538. Found: 405.0562. Anal. Calcd for $\mathrm{C}_{20} \mathrm{H}_{16} \mathrm{FeN}_{2} \mathrm{O}_{4}$ : C, 59.43; H, 3.99; $\mathrm{Fe}, 13.82$; N, 6.93; O, 15.83. Found C, 59.08; H, 3.71, N, 6.82.

\section{General procedure for the synthesis of ferrocene monosquaramides (18a-e)}

A 20-mL vial was charged with 2 (50 mg, $0.15 \mathrm{mmol}, 1$ equiv), the corresponding aminoester hydrochloride $(0.30 \mathrm{mmol}, 2$ equiv $)$, triethylamine $(0.30 \mathrm{mmol}, 2$ equiv), methanol $(4 \mathrm{~mL})$, and a magnetic stirrer. The vial was capped and the mixture was stirred until completion whereupon the solvent was removed under reduced pressure. The product was purified by simply trituration with hexanes and ethyl ether.

\section{General procedure for the synthesis of ferrocene bissquaramides (19a-e)}

A $20-\mathrm{mL}$ vial was charged with 3 (50 mg, $0.11 \mathrm{mmol}, 1$ equiv), the corresponding aminoester hydrochloride ( $0.43 \mathrm{mmol}, 4$ equiv), triethylamine ( $0.43 \mathrm{mmol}, 4$ equiv), methanol $(4 \mathrm{~mL})$, and a magnetic stirrer. The vial was capped and the mixture was stirred until completion whereupon the solvent was removed under reduced pressure. The product was purified by simply trituration with hexanes and ethyl ether.

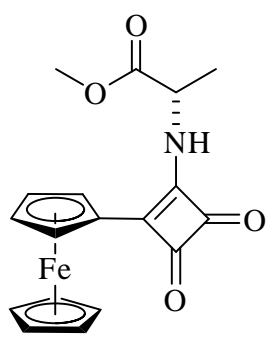

Methyl $(\boldsymbol{R})$-2-(3,4-dioxo-2-ferrocenylcyclobut-1-enylamino)propanoate (18a). According to GP. Red solid (43 mg, 79\%); TLC (4\% $\left.\mathrm{MeOH} / \mathrm{CH}_{2} \mathrm{Cl}_{2}, \mathrm{R}_{f}=0.7\right) ; \mathrm{mp}=170-171^{\circ} \mathrm{C}$. $[\alpha]^{20}{ }_{\mathrm{D}}=$ +27.7 (c = 0.01, $\left.\mathrm{CH}_{3} \mathrm{CN}\right)$. IR (KBr, cm $\left.{ }^{-1}\right): 3334(\mathrm{~m}), 2967(\mathrm{~m}), 1776(\mathrm{~s}), 1731$ (s), $1598(\mathrm{~s}), 1509$ 
(s), 1463 (s), 1333 (m), 1266 (m), 1122 (m), 1026 (m), $818(\mathrm{~m}) .{ }^{1} \mathrm{H}$ NMR (200 MHz, CD 3 CN): $\delta$ 6.8 (br s, 1H), 5.1 (q, J=7.2 Hz, 1H), 4.92 (br s, 2H), 4.6 (app d, J=1.6 Hz, 2H), 4.22 (s, 5H), 3.74 (s, 3H), 1.63 (d, J=7.4 Hz, 3H); ${ }^{13} \mathrm{C}$ NMR (50 MHz, CD $\left.{ }_{3} \mathrm{CN}\right): \delta$ 192.3, 190.0, 179.5, 173.2, 169.9, 72.8, 71.1, 68.9, 68.8, 53.7, 53.4, 18.8. Anal. Calcd for $\mathrm{C}_{18} \mathrm{H}_{17} \mathrm{FeNO}_{4}$ : C, 58.88; $\mathrm{H}, 4.67$; N, 3.81. Found: C, 58.79; H, 4.56; N, 3.79.

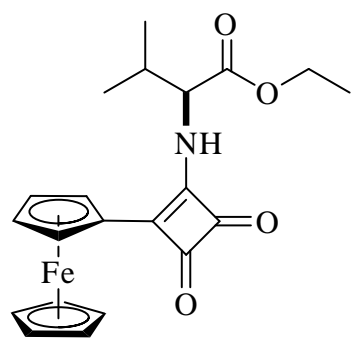

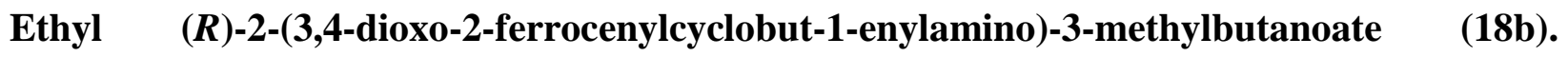
According to GP. Red solid (50 mg, 81\%); TLC (4\% $\mathrm{MeOH} / \mathrm{CH}_{2} \mathrm{Cl}_{2}, \mathrm{R}_{f}=0.7$ ); mp = 135-136 ${ }^{\circ} \mathrm{C} .[\alpha]^{20} \mathrm{D}=-4.11\left(\mathrm{c}=0.01, \mathrm{CH}_{3} \mathrm{CN}\right)$. IR $\left(\mathrm{KBr}, \mathrm{cm}^{-1}\right): 3309(\mathrm{~m}), 2967(\mathrm{~m}), 1780$ (i), $1736(\mathrm{~s})$, 1717 (s), 1597 (s) 1505 (s), 1465 (s), 1330 (m), 1222 (m), 1119 (m), 1041 (m), 1024 (m), 863 (m). ${ }^{1} \mathrm{H}$ NMR $\left(200 \mathrm{MHz}, \mathrm{CD}_{3} \mathrm{CN}\right): \delta 6.7(\mathrm{~d}, J=9.4 \mathrm{~Hz}, 1 \mathrm{H}), 4.9(\mathrm{br} \mathrm{s}, 2 \mathrm{H}), 4.8(\mathrm{t}, J=6.4 \mathrm{~Hz}$, $1 \mathrm{H}), 4.6$ (app t, $J=2 \mathrm{~Hz}, 2 \mathrm{H}), 4.2$ (q, overlapped, $2 \mathrm{H}), 4.2(\mathrm{~s}, 5 \mathrm{H}), 2.4(\mathrm{~m}, 1 \mathrm{H}), 1.3(\mathrm{t}, J=7 \mathrm{~Hz}$, $3 \mathrm{H}), 1.1(\mathrm{~d}, J=2 \mathrm{~Hz}, 6 \mathrm{H}) .{ }^{13} \mathrm{C} \mathrm{NMR}\left(50 \mathrm{MHz}, \mathrm{CD}_{3} \mathrm{CN}\right): \delta 192.3,179.5,171.6,170,72.7,71.1$, 70.6, 69.0, 68.8, 63.4, 62.5, 32.5, 19.3, 18.4, 14.6. Anal. Calcd for $\mathrm{C}_{21} \mathrm{H}_{23} \mathrm{FeNO}_{4}$ : C, 61.63; $\mathrm{H}$, 5.66; N, 3.42. Found: C, 61.71; H, 5.54; N, 3.49.

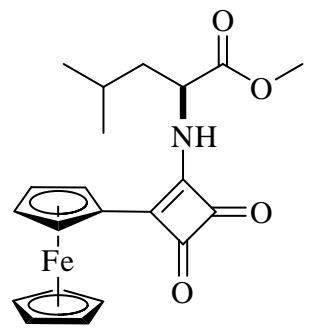

Methyl $(\boldsymbol{R})$-2-(3,4-dioxo-2-ferrocenylcyclobut-1-enylamino)-4-methylpentanoate $\quad(18 c)$. According to GP. Red solid (47 mg, 77\%); TLC (4\% MeOH/CH $\mathrm{CH}_{2}, \mathrm{R}_{f}=0.7$ ); $\mathrm{mp}=162-164$ ${ }^{\circ} \mathrm{C} .[\alpha]^{20} \mathrm{D}=-18.11\left(\mathrm{c}=0.01, \mathrm{CH}_{3} \mathrm{CN}\right)$; IR $\left(\mathrm{KBr}, \mathrm{cm}^{-1}\right): 3309(\mathrm{~m}), 2967(\mathrm{~m}), 1780(\mathrm{~s}), 1736(\mathrm{~s})$, 1717 (s), 1597 (s), 1505 (s), 1465 (s), 1330 (m), 1222 (m), 1119 (m), 1024 (m), 863 (m). ${ }^{1} \mathrm{H}$ NMR (200 MHz, DMSO- $\left.d_{6}\right): \delta 8.7$ (d, $\left.J=8.6 \mathrm{~Hz}, 1 \mathrm{H}\right), 5$. (app d, $\left.J=5 \mathrm{~Hz}, 2 \mathrm{H}\right), 5.0$ (q, J= $3 \mathrm{~Hz}$, $1 \mathrm{H}), 4.6(\operatorname{app~t}, J=1.8 \mathrm{~Hz}, 2 \mathrm{H}), 4.2(\mathrm{~s}, 5 \mathrm{H}), 3.7(\mathrm{~s}, 3 \mathrm{H}), 1.9(\mathrm{~m}, 1 \mathrm{H}), 1.7$ (app t, $J=5.2 \mathrm{~Hz}, 2 \mathrm{H})$, 0.97 (two d, $J=5.8 \mathrm{~Hz}, 6 \mathrm{H}$ ). ${ }^{13} \mathrm{C} \mathrm{NMR}\left(50 \mathrm{MHz}, \mathrm{CD}_{3} \mathrm{CN}\right.$ ): $\delta 192.3,189.9,179.5,173.1,170.2$, $72.8,71.1,70.5,69.0,68.7,56.5,53.3,41.5,25.6,23.4,21.3$. Anal. Calcd for $\mathrm{C}_{21} \mathrm{H}_{23} \mathrm{FeNO}_{4}$ : C, $61.63 ; \mathrm{H}, 5.66 ; \mathrm{N}, 3.42$. Found: C, 61.52; H, 5.45; N, 3.71. 


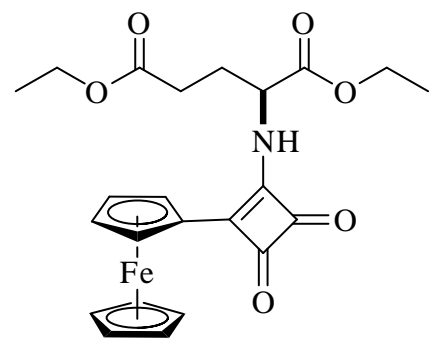

Diethyl (R)-2-(3,4-dioxo-2-ferrocenylcyclobut-1-enylamino)pentanedioate (18d). According to GP. Red solid (55 mg, 78\%); TLC (4\% $\left.\mathrm{MeOH} / \mathrm{CH}_{2} \mathrm{Cl}_{2}, \mathrm{R}_{f}=0.7\right)$; $\mathrm{mp}=131-132{ }^{\circ} \mathrm{C} .[\alpha]^{20}{ }_{\mathrm{D}}=-$ 26.04 (c=0.01, $\left.\mathrm{CH}_{3} \mathrm{CN}\right)$. IR (KBr, cm $\left.{ }^{-1}\right): 3193(\mathrm{~m}), 2980$ (m), 1776 (s), 1738 (s), 1738 (s), 1711 (s), 1582 (s), 1464 (s), 1227 (m), 1110 (m), 1026 (m), 820 (m). ${ }^{1} \mathrm{H}$ NMR (200 MHz, CD $\left.{ }_{3} \mathrm{CN}\right): \delta$ 6.9 (d, J=8.2 Hz, 1H), 4.9 (br s, $2 \mathrm{H}), 4.6$ (br s, $2 \mathrm{H}), 4.2$ (s, 5H), 4.2 (overlapped, 2H), 4.1 (q, J $=7 \mathrm{~Hz}, 2 \mathrm{H}), 2.56(\mathrm{t}, J=7.2 \mathrm{~Hz}, 2 \mathrm{H}), 2.3(\mathrm{t}, J=6.8 \mathrm{~Hz}, 1 \mathrm{H}), 2.1(\operatorname{app~q}, J=6.4 \mathrm{~Hz}, 2 \mathrm{H}), 1.3(\mathrm{~m}, J$ $=7 \mathrm{~Hz}, J=6.6 \mathrm{~Hz}, 6 \mathrm{H}) .{ }^{13} \mathrm{C} \mathrm{NMR}\left(50 \mathrm{MHz}, \mathrm{CD}_{3} \mathrm{CN}\right): \delta 192.2,190.0,179.8,173.7,171.8$, 170.2, 72.8, 71.1, 70.4, 68.9, 68.8, 62.8, 61.5, 57.4, 31.2, 28.0, 14.5. Anal. Calcd for $\mathrm{C}_{23} \mathrm{H}_{25} \mathrm{FeNO}_{6}$ : C, 59.12; H, 5.39; N, 3.00;. Found: C, 59.02; H, 5.51; N, 3.28.

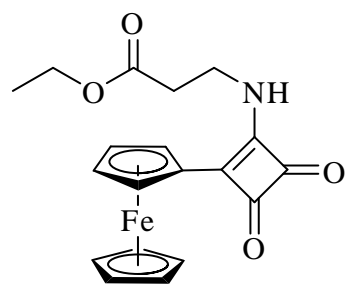

Ethyl 3-(3,4-dioxo-2-ferrocenylcyclobut-1-enylamino)propanoate (18e). According to GP. Red solid (53 mg, 94\%; TLC (4\% MeOH/ $\left.\mathrm{CH}_{2} \mathrm{Cl}_{2}, \mathrm{R}_{f}=0.5\right) ; \mathrm{mp}=154-155^{\circ} \mathrm{C}$; IR $\left(\mathrm{KBr}, \mathrm{cm}^{-1}\right)$ : 3161 (m), 2934 (m), 1777 (s), 1739 (s), 1708 (s)1580 (s), 1518 (s), 1433 (s), 1373 (s), 1265 (s),1105 (m),1043 (m), 840 (m). ${ }^{1} \mathrm{H}$ NMR (200 MHz, DMSO- $d_{6}$ ): $\delta 8.5$ ( br s, 1H), 5.0 (app t, $J$ $=2 \mathrm{~Hz}, 2 \mathrm{H}), 4.6(\mathrm{app} \mathrm{t}, J=2 \mathrm{~Hz}, 2 \mathrm{H}) 4.1(\mathrm{~s}, 5 \mathrm{H}), 4.1(\mathrm{q}, J=7.2 \mathrm{~Hz}, 2 \mathrm{H}), 3.9$ (q, $J=6.6 \mathrm{~Hz}, 2 \mathrm{H})$, $2.7(\mathrm{t}, J=6.4 \mathrm{~Hz}, 2 \mathrm{H}), 1.2(\mathrm{t}, J=7 \mathrm{~Hz}, 3 \mathrm{H}) .{ }^{13} \mathrm{C}$ NMR (50 MHz, DMSO- $\left.d_{6}\right): \delta 191.5,188.0$, 177.9, 170.6, 167.0, 71.2, 69.8, 69.6, 67.3, 60.0, 34.7, 15.1. Anal. Calcd for $\mathrm{C}_{19} \mathrm{H}_{19} \mathrm{FeNO}_{4}$ : C, 59.86; H, 5.02; N, 3.67. Found: C, 59.67; H, 4.13; N, 3.79. 


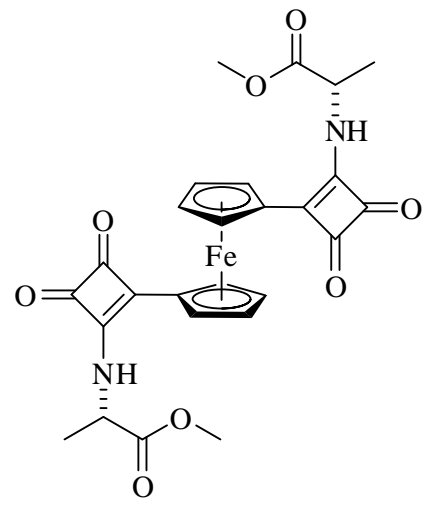

Dimethyl $\quad\left(2 R, 2^{\prime} R\right)-2,2^{\prime}$-[2,2'-(1,1'-ferrocenyl)bis(3,4-dioxocyclobut-1-ene-2,1-diyl)]bis(azanediyl) dipropanoate (19a). According to GP. Violet solid (33 mg, 57\%); TLC (4\% MeOH/ $\mathrm{CH}_{2} \mathrm{Cl}_{2}, \mathrm{R}_{f}$ $=0.5) ; \mathrm{mp}=270{ }^{\circ} \mathrm{C}$ (decomposed). $[\alpha]^{20}{ }_{\mathrm{D}}=+47.7\left(\mathrm{c}=0.01, \mathrm{CH}_{3} \mathrm{CN}\right) . \mathrm{IR}\left(\mathrm{KBr}, \mathrm{cm}^{-1}\right): 3329(\mathrm{~m})$, 1778 (s), 1728 (s), 1599 (s), 1467 (s), 1395 (m), 1339 (m), 1120 (m). ${ }^{1} \mathrm{H}$ NMR (200 MHz, DMSO- $\left.d_{6}\right): \delta 8.6(\mathrm{~d}, J=8.2 \mathrm{~Hz}, 2 \mathrm{H}), 4.8(\mathrm{q}, J=7.2 \mathrm{~Hz}, 2 \mathrm{H}$ ), 4.7 (br s, $4 \mathrm{H}), 4.6(\mathrm{br} \mathrm{s}, 4 \mathrm{H}), 3.6$ (s, $6 \mathrm{H}), 1.5(\mathrm{~d}, J=7.2 \mathrm{~Hz}, 6 \mathrm{H}) .{ }^{13} \mathrm{C}$ NMR (50 MHz, DMSO- $\left.d_{6}\right): \delta 192.3,191.6,191.0,174.4$, 172.1, 79.6, 74.2, 70.2, 69.5, 52.5, 22.4. Anal. Calcd for $\mathrm{C}_{26} \mathrm{H}_{24} \mathrm{FeN}_{2} \mathrm{O}_{8}: \mathrm{C}, 56.95 ; \mathrm{H}, 4.41 ; \mathrm{N}$, 5.11. Found: C, 56.69; H, 4.68; N, 5.37.

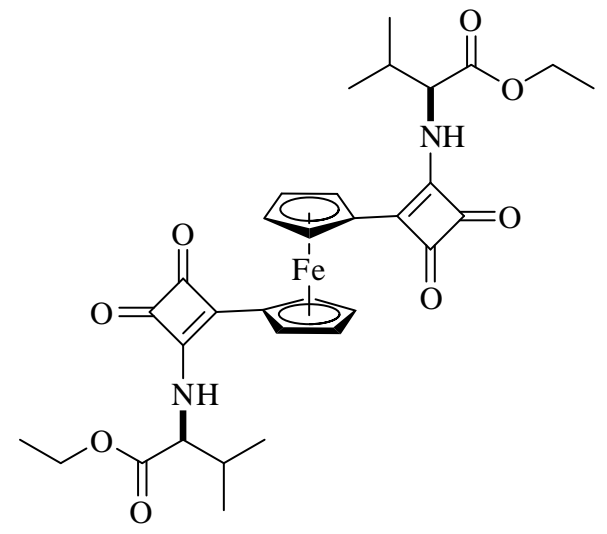

Diethyl (2R,2'R)-2,2'-(2,2'-(1,1'-ferrocenyl)bis(3,4-dioxocyclobut-1-ene-2,1-diyl))bis(azanediyl) bis(3-methylbutanoate) (19b). According to GP. Red solid (46 mg, 68\%); TLC (4\% $\left.\mathrm{MeOH} / \mathrm{CH}_{2} \mathrm{Cl}_{2}, \mathrm{R}_{f}=0.5\right) ; \mathrm{mp}=277^{\circ} \mathrm{C}$ (decomposed). $[\alpha]^{20}{ }_{\mathrm{D}}=-32.2\left(\mathrm{c}=0.01, \mathrm{CH}_{3} \mathrm{CN}\right.$ ); IR (KBr, cm$\left.{ }^{-1}\right): 3335(\mathrm{~m}), 2968(\mathrm{~m}), 1775$ (s), 1731 (s), 1598 (s) 1513 (s), 1462 (m), 1333 (m), 1039 $(\mathrm{m}), 842(\mathrm{~m}) .{ }^{1} \mathrm{H}$ NMR $\left(200 \mathrm{MHz}, \mathrm{CD}_{3} \mathrm{CN}\right): \delta 6.9(\mathrm{~d}, J=9 \mathrm{~Hz}, 2 \mathrm{H}), 4.9(\operatorname{app} \mathrm{d}, J=5.2 \mathrm{~Hz}, 4 \mathrm{H})$, $4.8(\mathrm{dd}, J=6 \mathrm{~Hz}, J=3.2 \mathrm{~Hz}, 2 \mathrm{H}), 4.6(\mathrm{br} \mathrm{s}, 4 \mathrm{H}), 4.3(\mathrm{q}, J=6.6 \mathrm{~Hz}, 4 \mathrm{H}), 2.3(\mathrm{~m}, 2 \mathrm{H}), 1.3(\mathrm{t}, J=$ $7 \mathrm{~Hz}, 6 \mathrm{H}), 1.1(\mathrm{dd}, J=3.4 \mathrm{~Hz}, 12 \mathrm{H}) .{ }^{13} \mathrm{C}$ NMR $\left(50 \mathrm{MHz}, \mathrm{DMSO}-d_{6}\right): \delta 191.7,188.1,178.1$, 170.3, 165.5, 73.0, 71.6, 69.6, 69.1, 62.1, 61.0, 30.6, 18.7, 18.5, 14.0. Anal. Calcd for $\mathrm{C}_{32} \mathrm{H}_{36} \mathrm{FeN}_{2} \mathrm{O}_{8}$ : C, 60.77; H, 5.74; N, 4.43. Found: C, 60.90; H, 5.68; N, 4.59. 


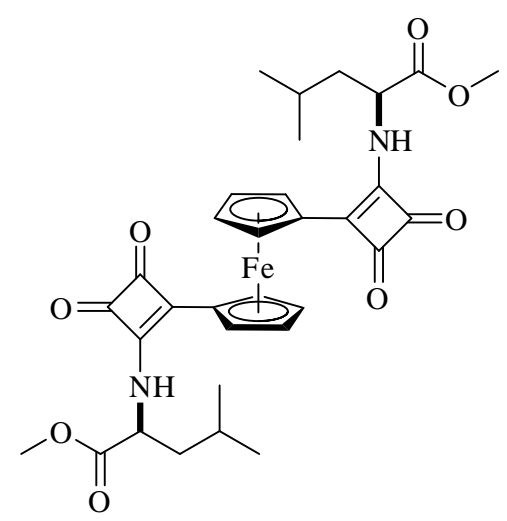

Dimethyl $\quad\left(2 R, 2^{\prime} R\right)-2,2^{\prime}-\left(2,2^{\prime}\right.$-(1,1'-ferrocenyl)bis(3,4-dioxocyclobut-1-ene-2,1-diyl))bis (azanediyl)bis(4-methylpentanoate) (19c). According to GP. Red solid (54 mg, 80\%); TLC (4\% MeOH/ $\mathrm{CH}_{2} \mathrm{Cl}_{2}, \mathrm{R}_{f}=0.4$ ); $\mathrm{mp}=235^{\circ} \mathrm{C}$ (decomposed). $[\alpha]^{20}{ }_{\mathrm{D}}=-33.39$ (c= 0.01, $\mathrm{CH}_{3} \mathrm{CN}$ ); IR $\left(\mathrm{KBr}, \mathrm{cm}^{-1}\right): 3263(\mathrm{~m}), 2958(\mathrm{~m}), 1775$ (s), 1746 (s), 1720 (s), 1589 (s), 1510 (s) 1460 (m)1213 (m), $830(\mathrm{~m}) .{ }^{1} \mathrm{H}$ NMR $\left(200 \mathrm{MHz}, \mathrm{CD}_{3} \mathrm{CN}\right): \delta 7.2(\mathrm{~d}, J=9.4 \mathrm{~Hz}, 2 \mathrm{H}), 5.0$ (q, 2H), 4.9 (d, 1.8 $\mathrm{Hz}, 4 \mathrm{H}), 4.6$ (d, 1.8H, 4H), 3.7 (s, 6H), 1.8 (t, J=10.4 Hz, 4H), $1.6(\mathrm{~m}, 1 \mathrm{H}), 1.0(\mathrm{t}, J=4.2 \mathrm{~Hz}, 12$ H). ${ }^{13} \mathrm{C}$ NMR $\left(50 \mathrm{MHz}, \mathrm{CD}_{3} \mathrm{CN}\right): \delta 192.48,190,180.12,173.28,167.49,74.82,74.61,70.25$, 69.8, 56.6, 53.3, 41.6, 25.5, 23.3, 21.5. Anal. Calcd for $\mathrm{C}_{32} \mathrm{H}_{36} \mathrm{FeN}_{2} \mathrm{O}_{8}: \mathrm{C}, 60.77$; H, 5.74; N, 4.43. Found: C, 60.90; H, 5.68; N, 4.58.

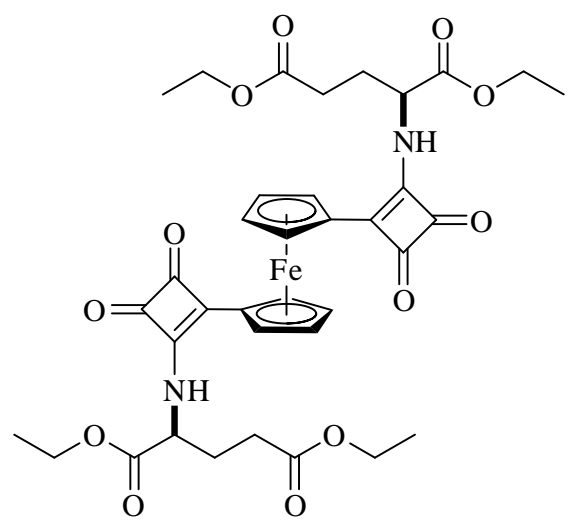

Tetraethyl $\quad\left(2 R, 2^{\prime} R\right)-2,2^{\prime}-\left(2,2^{\prime}\right.$-(1,1'-ferrocenyl)bis(3,4-dioxocyclobut-1-ene-2,1-diyl))bis (azanediyl)dipentanoate (19d). According to GP. Red solid (59 mg, 73\%); TLC (4\% $\left.\mathrm{MeOH} / \mathrm{CH}_{2} \mathrm{Cl}_{2}, \mathrm{R}_{f}=0.5\right) ; \mathrm{mp}=129-130{ }^{\circ} \mathrm{C} .[\alpha]^{20}{ }_{\mathrm{D}}=-59.07\left(\mathrm{c}=0.01, \mathrm{CH}_{3} \mathrm{CN}\right) ; \mathrm{IR}(\mathrm{KBr}): 3340$ (m), 2981 (m), 1777 (s), 1733 (s), 1601 (s), 1513 (s), 1461 (m), 1329 (m), 1027 (m), 927 (m). ${ }^{1} \mathrm{H}$ NMR $\left(200 \mathrm{MHz}, \mathrm{CD}_{3} \mathrm{CN}\right): 7.3(\mathrm{~d}, J=8.6 \mathrm{~Hz}, 2 \mathrm{H}), 4.9(\mathrm{~d}, J=8.6 \mathrm{~Hz}, 4 \mathrm{H}), 4.7(\mathrm{~d}, J=10.2 \mathrm{~Hz}$, $4 \mathrm{H}), 4.3(\mathrm{~m}, 8 \mathrm{H}), 2.6(\mathrm{t}, J=6.6 \mathrm{~Hz}, 4 \mathrm{H}), 2.4(\mathrm{~m}, 2 \mathrm{H}), 2.2(\mathrm{~m}, 4 \mathrm{H}), 1.3(\mathrm{~m}, 12 \mathrm{H}) .{ }^{13} \mathrm{C}$ NMR $(50$ $\left.\mathrm{MHz}, \mathrm{CD}_{3} \mathrm{CN}\right): \delta 192.4,189.9,180.3,173.9,171.8,74.7,74.4,72.7,70.2,69.7,62.9,61.5,57.6$, 31.2, 27.9, 14.5. Anal. Calcd for $\mathrm{C}_{36} \mathrm{H}_{40} \mathrm{FeN}_{2} \mathrm{O}_{12}$ : C, 57.76; H, 5.39; N, 3.74. Found: C, 57.91; H, $5.12 ; \mathrm{N}, 3.89$. 


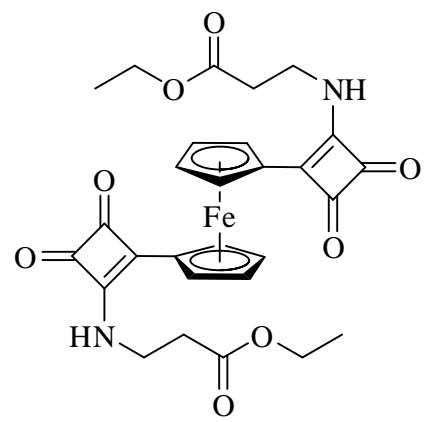

Diethyl $\quad 3,3^{\prime}$-(2,2'-(1,1'-ferrocenyl)bis(3,4-dioxocyclobut-1-ene-2,1-diyl))bis(azanediyl) dipropanoate (19e). According to GP. Red solid (55 mg, 88\%); TLC (4\% $\mathrm{MeOH} / \mathrm{CH}_{2} \mathrm{Cl}_{2}, \mathrm{R}_{f}=$ 0.3); $\mathrm{mp}=210-211^{\circ} \mathrm{C}$; IR (KBr): 3290 (m), 2985 (m), 1770 (s), 1729 (s), 1576 (s), 1513 (s), $1389(\mathrm{~m}), 1319(\mathrm{~m}), 1044(\mathrm{~m}), 924(\mathrm{~m}) .{ }^{1} \mathrm{H}$ NMR (200 MHz, DMSO- $\left.d_{6}\right): \delta 8.4$ (br s $\left.2 \mathrm{H}\right), 5.0$ (s, $4 \mathrm{H}), 4.6(\mathrm{~s}, 4 \mathrm{H}), 4.1(\mathrm{q}, J=7 \mathrm{~Hz}, 4 \mathrm{H}), 3.8(\mathrm{q}, J=6 \mathrm{~Hz}, 4 \mathrm{H}), 2.7(\mathrm{t}, J=7 \mathrm{~Hz}, 4 \mathrm{H}), 1.2(\mathrm{t}, J=7.2$ $\mathrm{Hz}, 6 \mathrm{H}) .{ }^{13} \mathrm{C}$ NMR $\left(50 \mathrm{MHz}, \mathrm{DMSO}-d_{6}\right): \delta 191.8,187.8,177.2,170.6,162.5,73.0,72.2,68.3$, 60.1, 34.5, 13.9. Anal. Calcd for $\mathrm{C}_{28} \mathrm{H}_{28} \mathrm{FeN}_{2} \mathrm{O}_{8}$ : C, 58.35; H, 4.90; N, 4.86. Found: C, 58.62; H, $4.98 ; \mathrm{N}, 4.79$.

\section{Acknowledgements}

This work was generously supported by CONACyT (CIAM program grant 2005-C02-51833/A1). F. V.-G. wishes to thank both CONACyT and CONCyTEG for a graduate scholarship.

\section{References}

1. (a) Ferrocenes: Homogeneous Catalysis, Organic Synthesis, Material Science; Togni, A., Hayashi, T. Eds.; VCH: New York, 1995. (b) Ferrocenes: ligands, materials and biomolecules; Stepnicka, P. Ed.; Wiley: West Sussex, 2008.

2. (a) Fouda, M. F. R.; Abd-Elzaher, M. M.; Abdelsamaia, R. A.; Labib, A. App. Organomet. Chem. 2007, 21, 613. (b) A. Allardyce, C. S.; Dorcier, A.; Scolaro, C.; Dyson, P. J. App. Organomet. Chem. 2005, 19, 1. (c) Fish, R. H.; Jaouen, G. Organometallics 2003, 22, 2166.

3. Dombrowski, K. E.; Baldwin, W.; Sheats, J. E. J. Organomet. Chem. 1986, 302, 281.

4. (a) van Staveren, D. R.; Metzler-Nolte, N. Chem. Rev. 2004, 104, 5931. (b) Azzaroni, O.; Alvarez, M.; Mir, M.; Yameen, B.; Knoll, W. J. Phys. Chem. C 2008, 112, 15850. (c) Moriuchi, T.; Nagai, T.; Fujiwara, T.; Honda, N.; Hirao, T. Heterocycles 2008, 76, 595. (d) Mir, M.; Alvarez, M.; Azzaroni, O.; Tiefenauer, L.; Knoll, W. Anal. Chem. 2008, 80, 6554. (e) Azzaroni, O.; Mir, M.; Alvarez, M.; Tiefenauer, L.; Knoll, W. Langmuir 2008, 24, 2878. (f) Lo, K. K.-W.; Lau, J. S.-Y.; Ng, D. C.-M.; Zhu, N. J. Chem. Soc., Dalton Trans. 2002, 8, 
1753. (g) Forrow, N. J.; Foulds, N. C.; Frew, J. E.; Law, J. T. Bioconjugate Chem. 2004, 15, 137.

5. (a) Top, S.; Tang, J.; Vessieres A.; Carrez, D.; Provot C.; Jaouen, G. Chem.Comm. 1996, 955. (b) Top, S.; Dauer, B.; Vaisserman, J.; Jaouen, G. J. Organomet. Chem. 1997, 541, 355.

6. Metzler-Nolte, N. Chimia 2007, 61, 736.

7. (a) Ge, D.; Levicky, R. Chem. Comm. 2010, 46, 7192. (b) Kraatz, H.-B.; Leek, D. M.; Houmam, A.; Enright, G. D.; Lusztyk, J.; Wayner, D. D. M. J. Organomet. Chem. 1999, 589, 38. (c) Xu, Y.; Kraatz, H.-B. Tetrahedron Lett. 2001, 42, 2601.

8. (a) Moriuchi, T.; Hirao, T. Acc. Chem. Res. 2010, 43, 1040. (b) Moriuchi, T.; Hirao, T. Top Organomet. Chem. 2006, 17, 143 and references therein. (c) Moriuchi, T.; Nagai, T.; Hirao, T. Org. Lett. 2006, 8, 31. (d) Seio, K.; Mizuta, M.; Terada, T.; Sekine, M. J. Org. Chem. 2005, 70, 10311. (e) de Hatten, X.; Cournia, Z.; Huc, I.; Smith, J. C.; Metzler-Nolte, N. Chem. Eur. J. 2007, 13, 8139. (f) de Hatten, X.; Bothe, E.; Merz, K.; Huc, I.; Metzler-Nolte, N. Eur. J. Inorg. Chem. 2008, 29, 4530. (g) Chowdhury, S.; Schantte, G.; Heinz-Bernhard, K. Angew. Chem. Int. Ed. 2008, 47, 7056. (h) Takeda, K.; Morita, T.; Kimura, S. J. Phys. Chem. B 2008, 112, 12840. (i) Moriuchi, T.; Nagai, T.; Fujiwara, T.; Honda, N.; Hirao, T. Heterocycles 2008, 76, 595-603. (j) Luo, X.; Lee, T. M.-H.; Hsing, I.-M. Anal. Chem. 2008, 80, 7341. (k) Wain, A. J.; Do, H. N. L.; Mandal, H. S.; Kraatz, H.-B.; Zhou, F. J. Phys. Chem. C 2008, 112, 14513. (1) Tebben, L.; Bussmann, K.; Hegemann, M.; Kehr, G.; Froehlich, R.; Erker, G. Organometallics 2008, 27, 4269. (m) Gasser, G.; Huesken, N.; Koester, S. D.; Metzler-Nolte, N. Chem. Comm. 2008, 31, 3675. (n) Scully, C. C. G.; Jensen, P.; Rutledge, P. J. J. Organomet. Chem. 2008, 693, 2869. (o) Fujimoto, K.; Kawai, H.; Amano, M.; Inouye, M. J. Org. Chem. 2008, 73, 5123. (p) Orlowski, G. A.; Chowdhury, S.; Kraatz, H.-B. Langmuir 2007, 23, 12765. (q) Miklan, Z.; Szabo, R.; Zsoldos-Mady, V.; Remenyi, J.; Banoczi, Z.; Hudecomposedz, F. Biopolymers 2007, 88, 108.

9. (a) Shinada, T.; Ishida, T.; Ohfune, Y. Tetrahedron Lett. 2005, 46, 311. (b) Shinada, T.; Nakagawa, Y.; Hayashi, K.; Corzo, G.; Nakajima, T.; Ohfune, Y. Amino Acids 2003, 24, 293. (c) Childers, W. E. Jr.; Abou-Gharbia, M. A.; Moyer, J. A.; Zaleska, M. M. Drugs Future 2002, 27, 633. (d) Sato, K.; Seio, K.; Sekine, M. J. Am. Chem. Soc. 2002, 124, 12715. 10. (a) Gassensmith, J. J.; Barr, L.; Baumes, J. M.; Paek, A.; Nguyen, A.; Smith, B. D. Org. Lett. 2008, 10, 3343. (b) Block, M. A. B.; Khan, A.; Hecht, S. J. Org. Chem. 2004, 69, 184. (c) Bueschel, M.; Ajayaghosh, A.; Arunkumar, E.; Daub, J. Org. Lett. 2003, 5, 2975. (d) Pham, W.; Weissleder, R.; Tung, C.-H. Tetrahedron Lett. 2003, 44, 3975. (e) Sreejith, S.; Carol, P.; Chithraa, P.; Ajayaghosh, A. J. Mat. Chem. 2008, 18, 264.

11. (a) Trost, B. M.; Thiel, O. R.; Tsui, H.-C. J. Am. Chem. Soc. 2003, 125, 13155. (b) Buchynskyy, A.; Kempin, U.; Vogel, S.; Hennig, L.; Findeisen, M.; Muller, D.; Giesa, S.; Knoll, H.; Welzel, P. Eur. J. Org. Chem. 2002, 7, 1149. (c) Peña-Cabrera, E.; Liebeskind, L. S. J. Org. Chem. 2002, 67, 1689. For reviews, see: (d) Clausen, C.; Wartchow, R.; Butenschon, H. Eur. J. Org. Chem. 2001, 93. 
12. Rehana B. Hossany, R. B.; Johnson, M. A.; Eniade, A. A.; Pinto, M. Bioorg. Med. Chem. 2004, 12, 3743.

13. Onaran, M. B.; Comeau, A. B.; Seto, C. T. J. Org. Chem. 2005, 70, 10792. For a more recent example see: Sejwal, P.; Han, Y.; Shah, A.; Luk, Y.-Y. Org. Lett. 2007, 9, 4897.

14. (a) Peña-Cabrera, E.; Aguilar-Aguilar, A.; Hernandez-Barba, A.; Rodriguez-Cendejas, C. G.; Liebeskind, L. S. Abstracts of Papers 20th Int. Conf. Organomet. Chem., Corfu, Greece, 712 July, 2002; p 194. (b) Aguilar-Aguilar, A.; Peña-Cabrera, E.; Liebeskind, L. S. Arkivoc 2004, (i), 156.

15. Zora, M.; Yucel, B.; Acikalin, S. Tetrahedron Lett. 2003, 44, 2237.

16. For other examples of squaramides, see: (a) Ramalingam, V.; Bhagirath, N.; Muthyala, R. S. J. Org. Chem. 2007, 72, 3976. (b) Rostami, A.; Colin, A.; Li, X. Y.; Chudzinski, M. G.; Lough, A. J.; Taylor, M. S. J. Org. Chem. 2010, 75, 3983. (c) Fu, N.; Baumes, J. M.; Arunkumar, E.; Noll, B. C.; Smith, B. D. J. Org. Chem. 2009, 74, 6462. (d) Muthyala, R. S.; Subramaniam, G.; Todaro, L. Org. Lett. 2004, 6, 4663. (e) Ramalingman, V.; Domaradzki, M. E.; Jang, S.; Muthyala, R. S. Org. Lett. 2008, 10, 3315. 